\title{
The Micro Radiation Environment Monitor (MuREM) and SSTL Radiation Monitor (SSTL RM) On TechDemoSat-1
}

\author{
B. Taylor, C. Underwood, A. Dyer, C. Ashton, S. Rason, J. Browning
}

\begin{abstract}
Two new, miniaturised scientific radiation monitoring payloads are presented prior to their first flight on the TechDemoSat-1 Spacecraft. They are capable of monitoring the space radiation environment and its effects on radiation sensitive devices. MuREM and SSTL RM carry RADFET dosimeters, dose rate sensitive photodiodes and PIN diode particle detectors. SSTL RM is also connected to external RADFET sensors placed around the spacecraft, whilst MuREM carries a radiation effects payload consisting of COTS devices which will be monitored whilst exposed to the space radiation environment.
\end{abstract}

Index Terms - ionising dose, LET, CEDEX, proton, radiation belts, radiation environment, radiation monitor, radiation effects, Singe Event Effects

\section{INTRODUCTION}

$\mathrm{T}$ he understanding of the radiation environment found in space and the effects it has on electronic components continues to be a critical concern for spacecraft designers and mission planners. With the development of more capable, miniaturised and innovative components, comes an increased risk of effects from radiation, both those that are well understood, and also newer effects, unique to new device technologies.

Ground testing of these devices has been shown to be of extensive, though limited value, due to the difficulty in simulating the space environment on the ground ${ }^{1,2}$, be it from high LET cosmic rays, high energy, high flux protons or high dose rates from energetic electrons. The aim of the MuREM payload is to provide in situ measurements of the radiation environment, whilst also providing data on the effect this environment has on modern, state of the art electronic devices.

Manuscript received September 16, 2011.

Dr. Ben Taylor is with the Surrey Space Centre, University of Surrey, Guildford, GU2 7XH, UK (phone: +44(0)1483 684711; fax: +44(0)1483 686021; e-mail: b.taylor@surrey.ac.uk).

Dr. Craig Underwood is with the Surrey Space Centre, University of Surrey, Guildford, GU2 7XH, UK (e-mail: c.underwood@ surrey.ac.uk).

Alex Dyer is with the Surrey Space Centre, University of Surrey, Guildford, GU2 7XH, UK (e-mail: a.dyer@surrey.ac.uk).

Chris Ashton is with the Surrey Space Centre, University of Surrey, Guildford, GU2 7XH, UK (e-mail: c.ashton@ surrey.ac.uk).

Samantha Rason is with Surrey Satellite Technology Ltd., Guildford, GU2 7YE (e-mail: s.rason@sstl.co.uk).

James Browning is with Surrey Satellite Technology Ltd., Guildford, GU2 7YE (e-mail: j.browning@sstl.co.uk).
The miniaturisation of electronic components allows this payload to itself be of small scale.

The SSTL Radiation Monitor is a cut down version of MuREM designed to measure the radiation environment only, and to provide real-time information on that environment to the spacecraft's housekeeping systems, so that appropriate mitigation strategies (increased memory washing and/or powering down of units) may be enacted.

Current spacecraft radiation design relies on the careful analysis of spacecraft structure and modelled environment, but does not routinely make use of in situ environment measurements. There is a growing understanding that whilst radiation environment models provide reasonable long term predictions, the actual environment encountered by spacecraft is far more dynamic. In addition, the modelling of the interaction of ionising radiation with the spacecraft shielding structure is not perfect and presents a margin of error ${ }^{3}$.

With the development of increasingly sophisticated spacecraft systems and payloads, the range of potential anomalies also increases. The diagnosis of these events can be complicated by a lack of in-situ data on the conditions surrounding them. Provision of radiation environment data allows the spacecraft operator to quickly determine whether any upset is due to radiation, and if so the nature of the interaction, whether it be due to high dose rates, heavy ions or high flux proton events. In addition, the use of long term total ionising dose monitoring allows mission planners to better judge the status of on board systems for possible mission extension.

The miniaturisation of electronic components allows MuREM and the SSTL Radiation Monitor to be of a small scale, whilst providing all relevant environment data using flight proven power, control and sensing systems. Both MuREM and the SSTL Radiation Monitor (RM) will be flown on the TechDemoSat-1 (TDS-1) spacecraft, with launch expected in 2012.

\section{INSTRUMENT OVERVIEW - MUREM}

\section{A. Instrument Description}

MuREM consists of three "PC104" style boards (approx $10 \mathrm{~cm} \times 10 \mathrm{~cm}$ ), and is sized to be mounted in a Cubesat configuration ${ }^{4}$. The unit as configured for TDS-1 uses a $10 \times 10 \times 4.5 \mathrm{~cm}$ housing with mounting feet and has a mass of approximately $0.5 \mathrm{~kg}$. MuREM makes use of RADFET solidstate dosimeters, a dose rate sensitive photo-diode and has two large area PIN diodes - one to measure proton flux and one to 
measure heavy ion flux and linear energy transfer (LET). The payload additionally carries a radiation effects board used to gather collateral data on devices exposed to the true environment found in space. Figure 1 shows the interior of the MuREM unit without the effects board in place. This board will sit above the detector board, which is visible in figure 1, showing the location of the large area diode detectors.

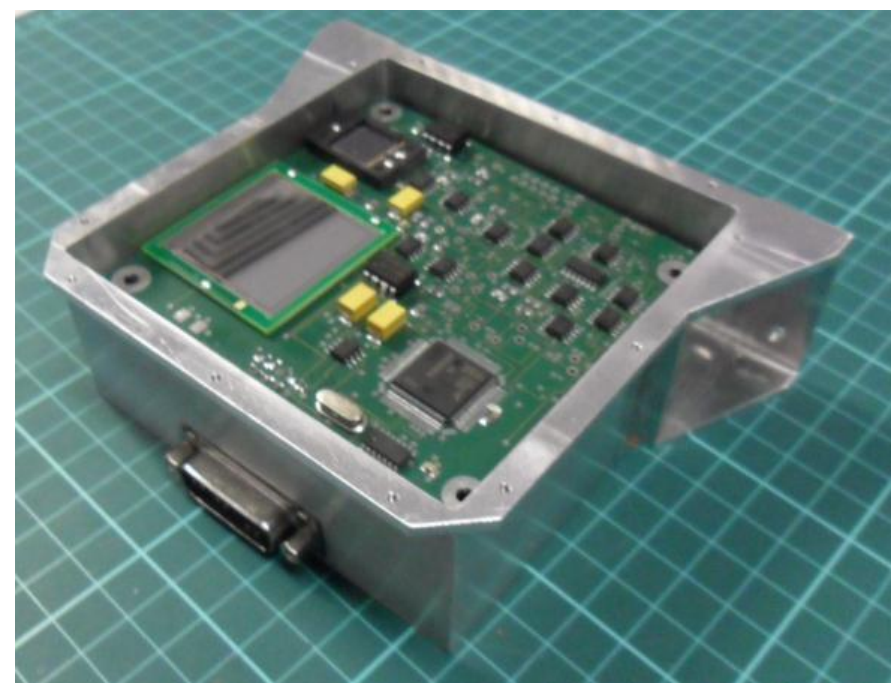

Fig. 1:The MuREM FM unit to be flown on TDS-1 showing the detector board with the two large area PIN diodes to the left of the image.

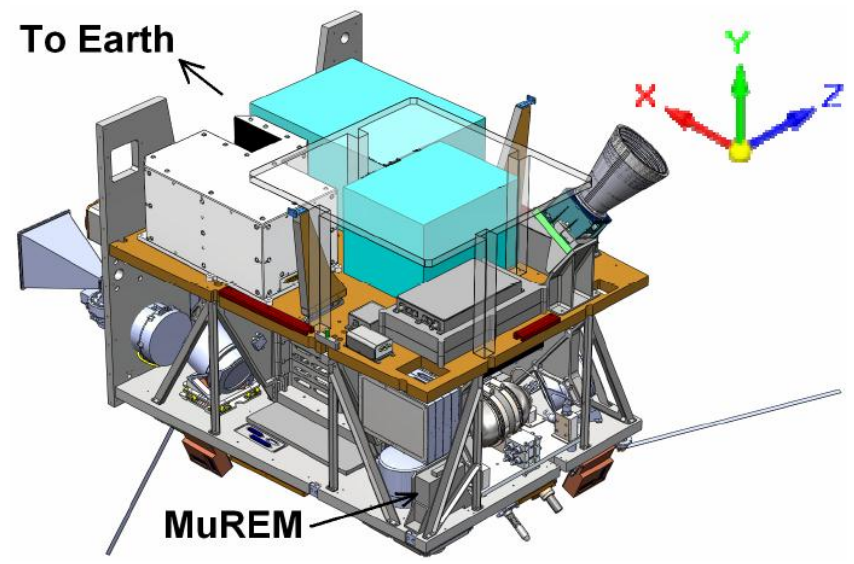

Fig. 2:Accomodation of the MuRM payload on the TDS-1 spacecraft

Each RADFET dosimeter consists of a pair of MOSFETs with a large gate oxide layer, increasing their response to total ionising dose (TID), which is detected by measuring the change to the gate threshold voltage. In measurement mode, one of the pair is held biased, whilst the other is held unbiased. In read mode, a fixed small current of approximately 6 microamps is passed through the MOSFET channel via the source and drain electrodes, and the required gate voltage is measured. The change in gate voltage varies linearly with accumulated dose and the rate of change of voltage with dose is bias dependent (more change for the biased FET). Unfortunately, the gate voltage is also strongly affected by temperature, however, by measuring the change in the biased and unbiased FET, the common temperature effect can be removed, leaving the dose-dependent change. A nearby temperature sensor provides supplementary temperature data, which can be used in case of failure of this detection methodology and the requirement to calibrate for dose. Thermal calibration of this system will also take place during Thermal Vacuum testing.

The output from the RADFET dosimeters takes the form of an analogue voltage, which is processed by an amplifier chain (to set gain and adjust voltage offset) before feeding into the C515C processor's 10-bit analogue-to-digital converter (ADC). In order to provide high resolution data, an adjustable voltage offset is applied to the analogue measurement circuit via a potentiometer voltage divider to maintain an output voltage range compatible with the ADC. In this way, the variation in the RADFET threshold voltage is allowed to be larger than the nominal voltage range of the ADC ( 0 to $4.096 \mathrm{~V}$ ) without having to unduly reduce the measurement circuit gain and thus reduce resolution. The approximate sensitivity of the biased RADFET is expected to be $\sim 1 \mathrm{mV}$ per cGy $(\mathrm{SiO} 2)$ of dose. Any fade should be less than $5 \%$ over the mission lifetime (up to 3 years). A telemetry code is included in the MuREM output providing a measure of the constant current used to measure the RADFETs. If this drifts over the mission lifetime, it can be used to determine any TID effects occurring within the RADFET read out electronics which might otherwise unduly skew dosimetry results. In the case of TDS this is unlikely to occur due to the low expected total dose.

REM RADFET sensors have been shown to exhibit the Enhanced Low Dose Rate Sensitivity (ELDRS) effect. ${ }^{5}$ The impact that this has on the total dose measurements during the mission can be quantified at dose rates down to $\operatorname{mrad}(\mathrm{Si}) \mathrm{s}^{-1}$ levels by the dose rate photodiode sensor. Further, it has been shown that RADFETs in a biased state show a greater ELDRS effect, post processing analysis of the biased and unbiased FETs should allow for this effect to be quantified.

The dose rate sensor consists of a metal-shielded silicon PIN photodiode connected to a very high transconductance gain amplifier of $10^{10} \mathrm{VA}^{-1}$ to act as a detector for the instantaneous ionisation dose-rate due to all ionising radiation (ions, electrons and Bremstrahhlung Xrays).

The "dark" current is a function of both ionising dose rate and temperature. However, as the diode has an approximately linear thermal response, the temperature effect can be calibrated out prior to flight, and the addition of a temperature sensor in MuREM means that, should the calibration change it can be re-calibrated in flight by noting the output variations with temperature outside of the main radiation zones (i.e. the South Atlantic Anomaly - SAA, and high latitude regions), where the dose-rate is solely due to Galactic Cosmic-Rays (GCRs) which is minimal.

Once calibrated, the remaining excess dark current signal is due to ionizing particles depositing energy into the detector and so monitoring this current provides a measure of the dose rate environment. This method was used on the CEDEX payload, flown on GIOVE-A with great success, and provided real time information on the ionizing dose-rate. A dose rate alarm threshold can be set at any level down to $\sim 0.001 \mathrm{cGy}(\mathrm{Si}) \mathrm{s}-1 \quad(10 \mathrm{pA})$ to provide warning of when the spacecraft is entering a high radiation environment (e.g. the SAA or over high latitudes during a solar particle event). 
The particle detectors consist of large area, $300 \mu \mathrm{m}$ thick PIN diodes whose outputs are run through CR-RC ${ }^{4}$ pulse shapers. The output pulse is held in a peak hold circuit whilst an ADC read is performed by the payload microcontroller, ensuring the true pulse peak is detected. A $3 \times 3 \mathrm{~cm}$ diode is used for detecting heavy ions and a $1 \times 1 \mathrm{~cm}$ diode is used for protons, owing to their relative fluxes. Particles travelling through the diodes will deposit energy dependent on their species and kinetic energy. Each particle event is logged in 16 logarithmically spaced energy deposition channels covering a LET range of approximately 2 to $20 \mathrm{MeV} \mathrm{cm} \mathrm{mg}^{-1}$ for the heavy ions. The proton detector has a different energy deposition threshold and range and is designed to detect protons with energies $>30 \mathrm{MeV}$. Each detector chain is capable of measuring up to 5,000 events per second, limited by the response time of software. Due to a greater noise level from the internal power system, and the instruments location within the spacecraft (Fig.2), the proton detector is less sensitive to the external proton environment than intended. Based on SRIM $^{7}$ simulations, the TDS MuREM Proton detector is only expected to generate an integral flux measurement of Protons with a LET range of approximately 0.1 to $5 \mathrm{MeV} \mathrm{cm} \mathrm{mg}^{-1}$. This issue is resolved for future use of the MuREM. The detector systems are equipped with an internally generated calibration pulse so that any drift in the detectors response with time or temperature can be monitored in orbit. The proton detector line is routed via an opto-coupler, whilst the heavy ion line is a direct $+5 \mathrm{~V}$ logic line. Any differential degradation of the proton calibration pulse compared to the heavy ion pulse would indicate damage to the opto-coupler, providing a qualitative indication of displacement damage. This is not expected to occur over the 3 year mission lifetime of TDS- 1 however.

MuREM operates on a $+5 \mathrm{~V}$ regulated power bus, with $+1.5 \mathrm{~V},+1.8 \mathrm{~V},+3.3 \mathrm{~V},-5 \mathrm{~V},+/-10 \mathrm{~V}$ lines generated internally. Outputs from all sensors are input into the 10-bit ADC of two internal C515C CAN controller devices. Two CAN bus addresses are used, with data from the particle detectors and radiation effects board delivered via CAN file-transfer protocol (FTP), with RADFET and dose rate diode data delivered as telemetry. The unit interfaces with the spacecraft via a single DA-26M connector containing all data and power links.

The payload is designed to operate in two modes. LOW POWER mode only operates the RADFET and dose rate diode sensors and the CAN interface. HIGH POWER mode additionally operates the radiation effects board and particle detector systems. Power draw for these two modes is approximately $0.3 \mathrm{~W}$ and $3 \mathrm{~W}$ respectively, dependent on the activity on the effects board. With the effects board switched off, the power draw in high power mode is $1.6 \mathrm{~W}$.

TID tests of the MuREM engineering model (EM) are planned with a Co-60 source in order to provide ground test data for the dosimetery systems on MuREM to improve confidence in the RADFET system. However, these are not critical before flight.

\section{B. Radiation Effects Payload}

The aim of the Effects Board on the TDS-1 MuREM is to assess the validity of using non-volatile memories such as
MRAM and FRAM in satellites and to test the ability of new mixed logic devices such as Texas Instruments TMS570 in the role of an intelligent supervisor.

As well as the TMS570 there is a PIC18F8680 from Microchip, this device will perform a range of operations, from the reading of and writing to the memory devices, the monitoring for single event effects (SEEs) in these devices and communicating with MuREM via the secondary C515C. The TMS570 is set up to communicate with the PIC18F8680 and monitor for current consumption increases in non-volatile memories. A current limiter is also used to prevent any single event functional interrupt (SEFI) or single event latch-up (SEL) from being damaging.

The use of a current limiter is important as it allows the system to assess and store information regarding the event before the power is cut from the board in order to power cycle the latched device. The non-volatile memories used on the effects board are the ferromagnetic MRAM module (MR0A08B) from Everspin and the ferroelectric FRAM module (FM22L16) from Ramtron. Each memory type will be used to create a memory block of $16 \times 1024$, as the MRAMs device is 8 bits wide, two devices will be wired as one to simulate a 16 bit wide device requiring six in total, while the FRAMs are 16 bits wide and so only three devices are needed. These memory modules will be constantly washed and monitored for SEEs with any events reported and corrected. These memory devices are both built upon CMOS technology, and so are susceptible to SEL, SEFI and single event transients (SETs). The MRAMs are regarded as inherently immune to SEUs due to their principle of operation, while the FRAM has been shown to be susceptible at energies as low as $2.7 \mathrm{MeV} \cdot \mathrm{mg}^{-1} \cdot \mathrm{cm}^{2}{ }^{6}$ The TMS570 has a wider capability in the space industry due to its high performance, internal mitigation and the use of mixed logic which not only allows the device to act as an intelligent supervisor capable of detecting the full range of SEEs found in commercial-off-theshelf (COTS) devices but also to replace many other devices and carry out their tasks. In order to test this capability, the TMS570 will also be washing its internal BRAM and using its flexray and CAN ports in a daisy chain.

The Effects board transmits data to the detector board microcontroller via UART serial link. 4 bytes are sent per SEU event, including memory address, bit pattern, with a reading from the ADC piggy-backing on the data. In addition, the effects board routinely sends a life sign data byte every 1-2 minutes so controllers can be sure the devices are functional. SEU data are entered into a register, allowing a maximum of 256 events to be registered every 5 minutes. If more than 256 events occur in a 5 minute interval, a counter for the device in question is incremented, rolling over after 65,536 counts. These data are transmitted by CANFTP along with the detector counts every 5 minutes.

A power switch allows the effects board to be isolated by telecommand in the event of a catastrophic failure, or to be power cycled in the event of a SEFI or SEL without switching off the entire MuREM payload.

A dual use for this board will be for it to be used as a generic ground test board, where memory devices can be easily replaced and tested using the fast wash rates and SEL protection. The boards will be designed so that all the control 
logic is located on one side of the board allowing it to be shielded during the device irradiation. This will allow the standardisation of any future memory tests carried out by the University of Surrey or SSTL while also decreasing the amount of time needed to prepare such an examination.

In support of the TDS-1 MuREM payload, heavy ion beam testing of the effects board is planned to provide a point of comparison between flight data and ground testing.

\section{INSTRUMENT OVERVIEW - SSTL RADIATION MONITOR}

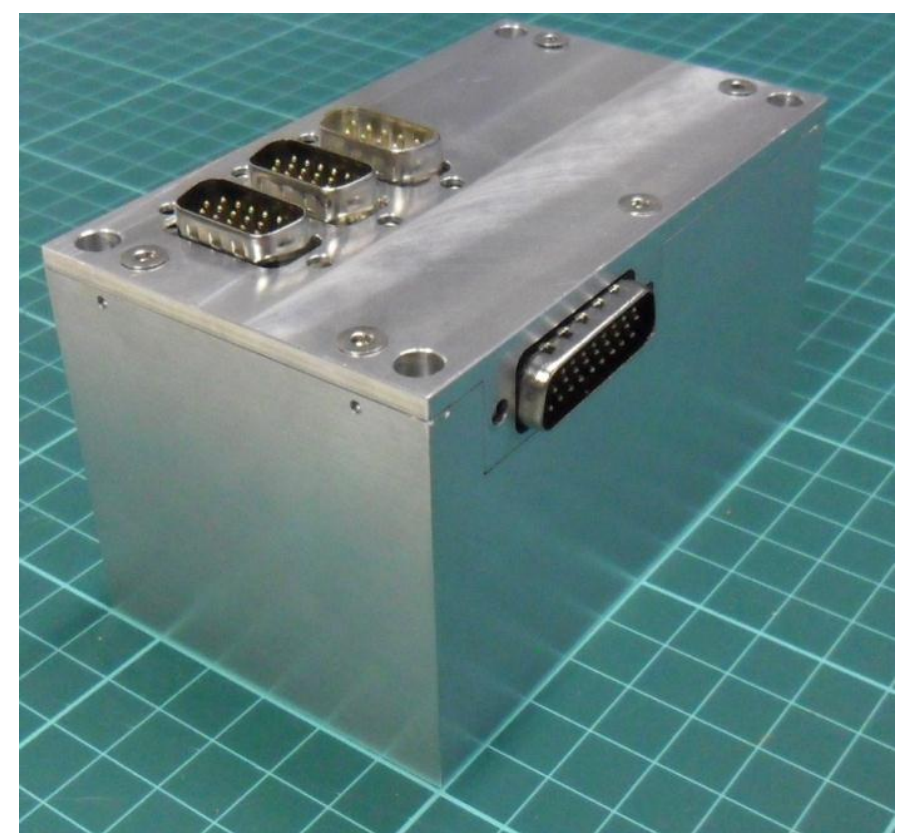

Fig. 3:SSTL RM EM showing three external RADFET ports and main 26 way connector

The SSTL RM is designed to be small enough to be routinely flown on a spacecraft to provide continuous environment monitoring as a standard. The SSTL RM is designed to fit in an envelope of $6 \times 6 \times 10 \mathrm{~cm}$ with a mass of approximately $400 \mathrm{~g}$. The unit contains five approximately credit-card sized PCBs, carrying all power, interface and sensing systems. The radiation monitor also has three DA-9 connectors to interface to three $1.5 \times 2.5 \mathrm{~cm}$ external TID sensor boards which can be positioned anywhere within the spacecraft containing RADFET dosimeters and temperature sensors. The sensors within the SSTL RM consist of an internal RADFET dosimeter, a single PIN diode particle detector and a photodiode configured to measure dose rate.

The particle detector consists of a $1 \times 1 \mathrm{~cm}, 300 \mu \mathrm{m}$ thick PIN diode whose output is run through a CR-RC ${ }^{4}$ pulse shaper. The detector provides a number of Linear Energy Transfer (LET) channels, with a counter provided for each. The thresholds for the TDS-1 SSTL RM are set such as to detect high energy but low LET protons, a 62 linearly spaced channel LET spectrum is generated covering the range 0.07 to $1 \mathrm{MeV}$ $\mathrm{cm}^{2} \mathrm{mg}^{-1}$, with the detector chain capable of measuring up to 10,000 events per second, limited by the speed of software processing. The LET range of the particle detector can be adjusted to cover a broader spectrum across mid range LET ions which could trigger soft Single Event Effects (SEE) and high LET particles which can be responsible for hard SEE.

As many systems are much more susceptible to TID and SEE whilst switched on, the SSTL RM can generate real time telemetry channels from the dose rate diode and particle detector to be monitored by either the spacecraft on-board computer (OBC) or a ground controller for quick action. A threshold level could be set on the OBC for each of these channels to switch off sensitive payloads within seconds of the onset of a severe radiation event, thus extending the lifetime of the spacecraft, and potentially protecting payloads or the spacecraft the spacecraft itself from failure.

The SSTL RM operates on a $28 \mathrm{~V}$ unregulated power bus, with $+/-5 \mathrm{~V}$ and $+/-10 \mathrm{~V}$ lines generated internally after filtering. Outputs from all sensors are input into the 10-bit ADC of two internal C515C CAN controller devices. Two CAN addresses are used, with 256 LET spectrum data delivered via CAN FTP and RADFET, dose rate diode and three-channel particle detector data delivered as telemetry. The unit interfaces with the spacecraft via a DA-26M connector containing all data and power links.

The SSTL RM can also be configured to run on a $+5 \mathrm{~V}$ regulated power line and can also make use of RS-232 data links.

The SSTL RM has been designed to accommodate an effects board if required. The detector microcontroller has a dedicated serial link line which can be connected to an effects board, along with an analogue and digital ground and a $+5 \mathrm{~V}$ power supply. The TDS-1 SSTL RM has an internal volume capable of housing an extra board.

As with MuREM, eventual TID tests are planned for the SSTL RM EM.

\section{HERITAGE}

The development of MuREM and the SSTL RM builds on the University of Surrey's two decades long experience with building space environment payloads which have flown on SSTL spacecraft.

The SSTL RM was conceived during the development of the MuREM radiation monitor at the University of Surrey, where the benefits of continuous, spacecraft level radiation monitoring were indentified. As such, much of the SSTL RM is based on the design work and heritage of the MuREM payload, with an increased focus on providing engineering data over the long term to mission planning, and in real time to the spacecraft $\mathrm{OBC}$ and spacecraft operators.

Both MuREM and the SSTL RM can trace their heritage back to the Cosmic Ray Experiment (CRE) payload developed at the University of Surrey and flown on the KITSAT-1 (1992) and PoSAT-1 (1993) spacecraft. These provided a virtually continuous record of proton and heavy-ion fluxes in $800 \mathrm{~km}$ Sun-synchronous orbit over a complete solar cycle. The CREs each contained a Total Dose Experiment (TDE) that measures the accumulated radiation dose at various locations in the spacecraft by means of RADFETs. The same circuit designs have been updated for use in the MuREM payload. The Cosmic-Particle Experiment (CPE) used in the CRE is also a fore-runner of the particle detector systems in MuREM. 


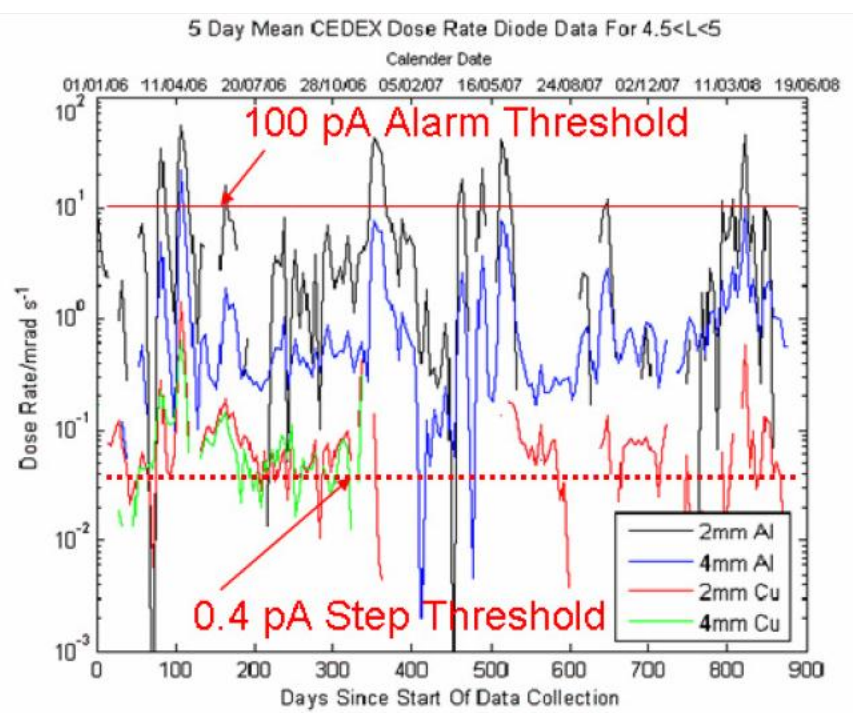

Fig. 4: GIOVE-A CEDEX photodiode dose-rate data for 880 days since launch for McIlwain L-shell 4.5<L<5.

In 1998, SSC developed a new miniaturised version of the CRE payload, known as CEDEX. This flew on TiungSAT-1 in 2000. This instrument formed the basis for the enhanced CEDEX instrument which was flown on ESA's GIOVE-A spacecraft launched in 2005. CEDEX also carries the experimental version of the dose-rate detectors used on MuREM which comprise small PIN diodes configured to give a direct photo current output. The diodes are placed behind domes of aluminium $(2 \mathrm{~mm}$ and $4 \mathrm{~mm}$ ) and copper $(2 \mathrm{~mm}$ and $4 \mathrm{~mm}$ ) to give a representative series of measurements of the dose-rate inside the spacecraft at different shielding depths. Experience with the CRE and CEDEX payloads has driven the design of the MuREM payload.

The results from GIOVE-A indicate that a 100pA photocurrent makes a good threshold for an automatic realtime severe radiation environment alarm, corresponding to a dose-rate of $0.01 \mathrm{cGy}(\mathrm{Si}) \mathrm{s}-1$ (i.e. $10 \operatorname{millirad}(\mathrm{Si})$ per second) Fig. 4.

The University of Surrey and SSTL have pioneered the use of COTS components on spacecraft, advising numerous bodies on the robust use of these devices in a space environment.

\section{MOTIVATION AND FUTURE APPLICATIONS}

\section{A. TechDemoSat-1 Mission}

The first version of the MuREM will be flown on the TechDemoSat-1 spacecraft. TDS-1 is a flagship mission by the UK Space Agency to demonstrate new technologies and methodologies for scientific and commercial applications on board a dedicated spacecraft. The spacecraft is being built by SSTL and will carry a range of payloads, including environment monitors, allowing for a high level of crosscalibration.

TDS-1 is being targeted at a launch in 2012 for a three year nominal mission into a $635 \mathrm{~km}, 98^{\circ}$ inclination sunsynchronous orbit allowing payloads to sample the South
Atlantic Anomaly and the polar horns of the outer electron belt.

MuREM and SSTL RM will be mounted internally on the TDS-1 spacecraft, and therefore a sector shielding analysis will be required to determine the shielding seen by the radiation instrumentation. For this paper, shielding of $5 \mathrm{~mm}$ Aluminium equivalent is assumed, including the $1.5 \mathrm{~mm} \mathrm{Al}$ unit housing. A full sector shielding model of the spacecraft will be simulated once the spacecraft is ready for launch. A Monte Carlo simulation will also be conducted to investigate the secondary particle population and its contribution to the detected fluxes.

Mean $>30 \mathrm{MeV}$ trapped proton fluxes are expected to be $\sim 40 \mathrm{~cm}^{-2} \mathrm{~s}^{-1}$, peaking at $\sim 3,000 \mathrm{~cm}^{-2} \mathrm{~s}^{-1}$ from AP-8 predictions. It is expected that SEEs detected on the effects board will be centred around the SAA, with secondary populations at higher latitudes due to heavy ions. If this pattern is detected, it will demonstrate SEE proton sensitivity in the devices under test. SEEs have been correlated to the SAA using past University of Surrey Radiation payloads, such as the CRE on KITSAT. ${ }^{8}$

The quiet time LET spectrum is not expected to vary greatly from the standard distribution observed in LEO, however significant solar particle events can add a significant mid LET component to this population which should be clear in the data.

The Total Ionising Dose received within the MuREM unit is expected to be on the order of $1 \mathrm{krad}$ for the 3 year mission, approximately evenly split between trapped protons, trapped electrons with associated bremsstrahlung and solar protons. Typical dose rates are expected to be very low, with occasional peaks due to the passage of the spacecraft through the SAA. Dose rates will also increase during the passage through the polar horns of the outer electron belt, however due to the level of shielding on the spacecraft this is not expected to be detectable by the MuREM dose rate photodiode sensor.

\section{B. Future Applications}

MuREM is designed to fit a Cubesat configuration for future low cost re-flight, allowing for responsive testing of new device technologies via the radiation effects board. This effects board is optional, and the payload may be flown using two boards as a pure radiation monitor.

In the future, it is expected that MuREM could fly in a CubeSat format with a series of different radiation effects test boards to act as an in-orbit verification system for the radiation performance of new devices - particularly commercial-off-the-shelf (COTS) devices. Surrey Space Centre (SSC) and SSTL have developed a 3U Cubesat technology demonstration bus as part of the STRaND project which would be a suitable vehicle for such a dedicated radiation environment and effects mission (Fig. 5).

Surrey Satellite Technology (SSTL) satellites are characterised by the extensive use of state-of-the-art commercial-off-the-shelf (COTS) micro-electronics to achieve complex functionality within very tight volume, mass and financial constraints. Each new generation of spacecraft has comprised bus sub-systems and payloads of increasing sophistication, utilising the many benefits of COTS devices namely their low cost, ready availability, high performance, 
low power and high packing density. SSTL's commercial and technical success shows that COTS devices can be used effectively in space, provided that care is taken in their procurement, handling and testing, and ensuring that sufficient thought is given to the spacecraft systems design. Because the radiation environment is such a major factor in determining the reliability of COTS devices in space, particular attention has been paid to monitoring this environment inside the spacecraft, and to analysing its effects through the use of SEEparticle detecting instruments and solid-state dosimeters. Over 20 years of scientific measurements have lead to a comprehensive set of radiation environment and effects data on COTS devices actually operating over an extended period in orbit. There is now the need for a more engineering focussed monitor; to establish confidence in new technology without much cost to power, mass and space.

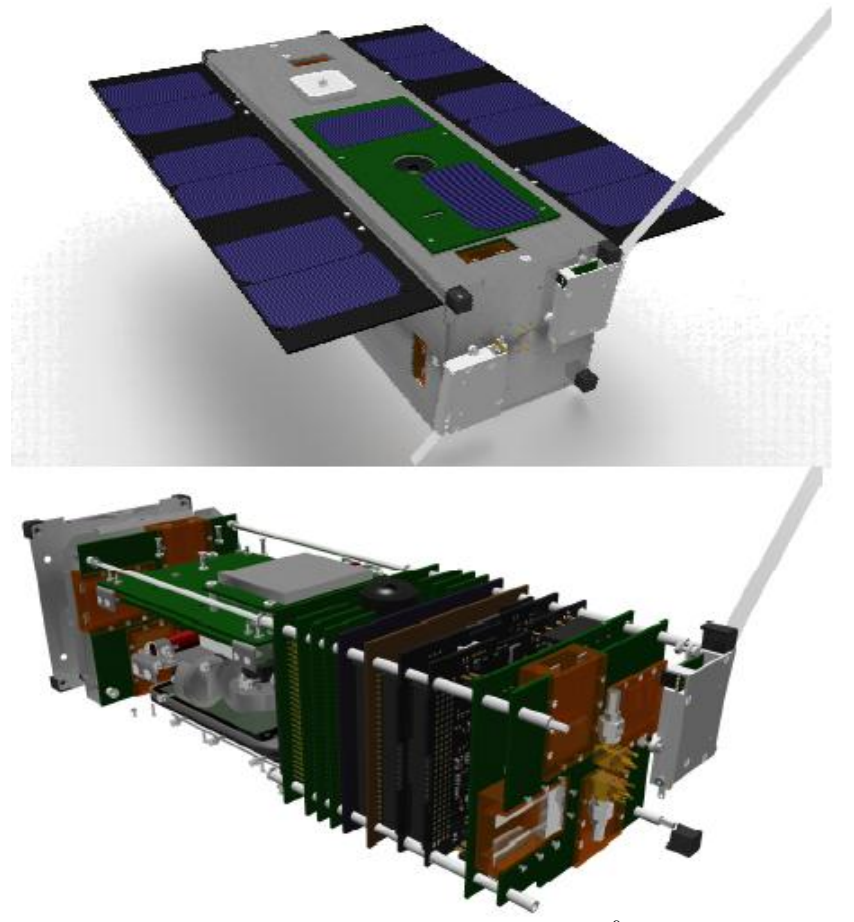

Fig. 5: Rendered images of the STRaND-1 3U Cubesat ${ }^{9}$

SSTL RM will fly on TechDemoSat and is base-lined for three further missions. It is intended that the SSTL RM will fly on every future mission.

SSTL is interested in the RM to:

- Reduce uncertainties from modelling by correlating sector shielding analysis models

- Qualify the company's RHA approach

- Improve flight heritage knowledge using in-flight data

- Aid with shielding design for TID sensitive devices;

- Improve spacecraft efficiency (e.g. reduce memory scrubbing rate in quiet environments);

- Gain confidence in cutting edge technology; allowing more efficient spacecraft design and avoiding component obsolescence

The SSTL RM can be reconfigured to carry a small radiation effects board similar to the one developed for MuREM allowing a small sample of a single device to be flown within the radiation monitor, providing flight heritage

for new, untested parts. In addition, the option of replacing one of the external RADFET sensors with an externally mounted miniaturised electron monitor is being investigated. This would be particularly beneficial in potential GEO spacecraft due to the severe electron environment at these altitudes. Likewise, other secondary sensors could be equipped on these ports, such as a Displacement Damage (DD) monitor consisting of known DD sensitive devices.

The dose sensors can be reconfigured in both MuREM and SSTL RM to increase or decrease sensitivity to provide an appropriate dynamic range for the expected environment.

\section{ACKNOWLEDGEMENTS}

The authors would like to thank BNSC, CNES, DERA (now known as QinetiQ), EPSRC, ESA-ESTEC, KAIST, the PoSAT Consortium, and Astronautic Technology (M) SDN. BHD. (ATSB) Malaysia and SSTL, for their support of this work over many years.

MuREM is an internally funded project of the University of Surrey/ Surrey Space Centre, however, particular thanks go to EADS-Astrium BV who sponsor the PhD work of Alex Dyer and Chris Ashton on Space Radiation Effects Analysis and Mitigation in Modern COTS Devices on behalf of SSTL Work which includes the development of the MuREM Radiation Effects Board.

\section{REFERENCES}

\section{]}

8]

9]

S. Kenyon, C. Bridges, et al, "STRaND-1: Use of a $\$ 500$
Smartphone as the central avionics of a nanosatellite", IAC-
11-B4.B6.8, $62^{\text {nd }}$ International Astronautical Congress, Cape
Town, SA, 2011 .

E. G. Stassinopoulos, "Shortcomings in Ground Testing, Environment simulations, and Performance Predictions for Space Applications," RADECS 1991 Proceedings, pp.3-16; R. Koga, "Single Event Effect Ground test issues," IEEE TNS, vol. 43, no 2, pp. 661-670, April 1996; R. Lindberg, G. Santin, H. Evans, E. Daly, P. Nieminen, "Detailed Comparison of Monte Carlo and Sector-Shielding Analyses for Space Applications" IEEE Transactions on Nuclear Science, vol. 53, issue 6, pp. 3745-3749, 2006; Cubesat Design Specification, Revision 12, The Cubesat Program, Caly Poly, SLO, 2009;

S.J. Kim, J. Seon, K.W. Min, Y.H. Shin, W. Choe,

"Enhanced low dose rate sensitivity (ELDRS) observed in RADFET sensor", Proceedings of the $7^{\text {th }}$ European Conference on Radiation and Its Effects on Components and Systems, RADECS 2003, pp. 669-671, 2003.

M. Campola, J. Howard, H. Kim, A. Phan, "Single Event Effects (SEE) Testing of the FM22L16 Ferroelectric Random Access Memory", NASA/GSFC Radiation Effects \& Analysis, 2007.

SRIM downloaded and accessed from www.srim.org, J. Ziegler, 2011.

C.I. Underwood, M.K. Oldfield, "Observed RadiationInduced Degradation of Commercial-Off-The-Shelf (COTS) Devices Operating in Low-Earth Orbit", IEEE TNS, vol. 45, No. 6, 1998, pp. 2737-2744 Town, SA, 2011. 